\title{
Guided Exploration for Coordinated Autonomous Soaring Flight
}

\author{
Kwok Cheng* \\ Jack W. Langelaan ${ }^{\dagger}$ \\ The Pennsylvania State University, University Park, PA 16802, USA
}

\begin{abstract}
An approach to guide cooperative wind field mapping for autonomous soaring is presented. The environment is discretized into a grid and a Kalman filter is used to estimate vertical wind speed in each cell. Exploration is driven by uncertainty in the vertical wind speed estimate and by the relative likelihood that a thermal will occur in a given cell. This relative likelihood is computed based on solar incidence, computed from a digital elevation map of terrain (obtained from US Geological Survey) and the position of the sun. An exploration priority function is computed for each cell, and mapping is guided by this exploration priority. The effectiveness of this approach is evaluated by using Monte Carlo simulation with different flock sizes, topographic elevation models and different level of altitude floors that triggers immediate energy exploitation mode. Simulation result indicates an overall improvement in endurance while priori information is precise. With high uncertainty in thermal distribution, a high altitude floor prevents performance loss in guided exploration. Final simulation result obtained using a commercially available high fidelity simulator demonstrates feasibility of the cooperative mapping and guided exploration approach.
\end{abstract}

\section{Introduction}

Rapid advancements in technology have accelerated the development of uninhabited aerial vehicles (UAVs) with more capabilities, which have expanded their application into areas including surveillance, communications relays, environmental monitoring and even combat. High altitude, long endurance (HALE) and medium altitude long endurance (MALE) UAVs such as Global Hawk and Predator have demonstrated outstanding performance for military applications as a result of the advanced sensors providing high resolution images. The combination of large fuel payload capacity and aerodynamics efficiency enables persistent surveillance; flight times of tens of hour has been reported for Predator and Global Hawk.

However, flight at high altitudes can lead to lack of target visibility due to clouds. Flight below cloud base (in the atmospheric boundary layer) would allow continuous surveillance even on cloudy days, but large vehicles become vulnerable to detection or destruction from ground-based threats. The use of small unmanned air vehicles (SUAS) would enable persistent surveillance from within the atmospheric boundary layer.

The small size of hand-launchable UAVs makes them inherently more stealthy than their larger counterparts. However, range and endurance of SUAS remains a problem: small payload size limits both the sensing payload and the ability to carry fuel or batteries, and the lower Reynolds numbers associated with sUAS results in lower overall aerodynamic efficiency. Currently, flight times of only one to one and a half hours are possible with hand launchable aircraft, making persistent surveillance difficult.

However, significant energy is available from the atmosphere. Large birds such as hawks and vultures (which are similar in size and aerodynamic performance to SUAS) routinely employ soaring flight to traverse hundreds of kilometers over several hours without flapping wings, and human sailplane pilots (both full scale and radio-controlled) routinely soar. The capability to soar autonomously would thus have a significant impact on the operational utility of small unmanned aircraft.

\footnotetext{
* Graduate Student, Department of Aerospace Engineering, Student Member AIAA.

${ }^{\dagger}$ Associate Professor, Department of Aerospace Engineering, Associate Fellow AIAA.
} 
There has been a significant amount of research relating to autonomous soaring conducted over the past few years. Recent works including simulation results of thermal flight are reported by Allen $(2005)^{1}$ and flight test results are presented in Allen (2007). ${ }^{2}$ Autonomous thermal soaring has also been addressed by Edwards. ${ }^{3}$ Research by Andersson and Kaminer has focused on flight control for thermal soaring. ${ }^{4,5}$ Soaring by manned sailplanes has also been studied extensively, with several authors addressing the optimal static soaring trajectory problem in the context of soaring competition. The MacCready problem, ${ }^{6,7}$ the final glide problem, ${ }^{8}$ and "Dolphin" flight along regions of alternating lift and $\operatorname{sink}^{9-11}$ all address optimal static soaring including optimal speed to fly between thermals of known strength. de Jong ${ }^{12}$ describes a geometric approach to trajectory optimization. Most of this research is limited by known lift distribution (e.g. sinusoidally varying lift ${ }^{13}$ or "square wave" lift $^{14}$ ) and generally do not consider the effects of horizontal wind components.

This earlier research has demonstrated the feasibility and utility of autonomous soaring for improving range and endurance of SUAS. Most autonomous soaring research has focused on exploiting thermals (columns of warm, rising air triggered by uneven solar heating of the ground combined with atmospheric instability), and because thermal formation is inherently non-deterministic typically autonomous thermal soaring has relied on aircraft randomly finding a thermal to exploit (essentially counting on luck to extend endurance). Research by Depenbusch has shown that the availability of a map can greatly improve overall performance, ${ }^{15}$ and also showed that mapping is improved as flock size in increased. However, the mapping in that work was driven by uncertainty in the map: in essence an aircraft explored regions where uncertainty in the map was high. This leads to a well-explored space, but can lead to cases where resources are wasted exploring regions where thermals are unlikely to be found. Note that energy can be harvested from predictable, deterministic phenomena such as ridge lift and wave ${ }^{16}$ the focus of this paper is on improving performance in stochastic environments.

The problem at hand is to develop a means to guide exploration behavior to focus on areas that are more likely to contain thermals. Several factors are known to affect thermal formation: solar incidence angle, terrain albedo, vegetation, and soil moisture content all play a role, with solar incidence angle and albedo having the largest influence. Atmospheric parameters such as lapse rate are important, but these typically vary over a larger scale than terrain-dependent parameters.

The research presented in this paper combines the mapping algorithm developed earlier (and described in detail in Depenbusch ${ }^{15}$ ) with a priori information of regions where thermal formation is more likely to guide exploration. Predictive tools such as BLIPMAPS (used to aid flight planning for sailplane and hangglider pilots) provide information on a coarse grid (roughly kilometer-sized), which is too large for the small unmanned aircraft considered here.

The remainder of this paper describes the simulation environment in Section III. Section A provides background information on thermal updrafts estimation. Section B defines the aircraft model and low-level controller. Section C and IV describe aircraft behaviors determination and guided exploration approach. Section V presents Monte Carlo simulation result for performance validation. Section VI demonstrates guided exploration in a commercially available high fidelity multi-player soaring flight simulator (Silent Wings). Section VII presents concluding remarks.

\section{Problem Statement and Background}

This research ultimately seeks to enable persistent surveillance via atmospheric energy harvesting. A flock of small aircraft cooperates to ensure that at least one vehicle maintains surveillance of the desired target; the remaining aircraft are free to harvest energy from the atmosphere or map the environment in the vicinity (within a couple of kilometers) of the surveillance target.

In earlier work a means of cooperatively mapping the environment using an approach derived from occupancy grid mapping is described. ${ }^{15}$ For completeness the mapping algorithm and flight behaviors to enable mapping are briefly outlined here.

\section{A. Mapping energy available in the atmosphere}

The environment is discretized into a grid of equally spaced cells, with cell size determined by the coordinated turn radius $R$ of the aircraft in typical thermalling flight conditions (roughly 25 meters for the RnR Products SB-XC radio-controlled sailplane). A smaller grid spacing provides higher resolution in energy map but it 
introduces higher level of correlation between cells for updraft estimation. With the grid of 25 meters spacing, aircraft is able to track waypoint to nearby cells and to thermal without crossing to surrounding cell. The presence of thermal can then be located at current aircraft location.

The mapping algorithm is implemented as an array of 1-D scalar Kalman filters. Each cell is associated with an estimated of vertical wind speed $\hat{w}_{z, i j}$ and error covariance of the estimated wind speed $P_{i j}$. The error covariance indicates the level of confidence in wind estimation associated with each cell. Small values in error covariance indicates confidence in wind speed estimation. Conversely, high value in error covariance indicates uncertainty in estimated wind speed, which requires another measurement to be taken later.

The vertical wind speed in a cell is assumed to be independent of surrounding cells. Note that this assumption is not strictly accurate (since a thermal can be larger than a single grid cell) but ignoring correlation between cells is conservative.

The dynamics of vertical wind speed in a cell is assumed to be linear with zero-mean Gaussian noise:

$$
\begin{aligned}
w_{z, i j, k+1} & =a w_{z, i j, k}+w_{k} \\
w_{k} & \sim \mathcal{N}(0, Q)
\end{aligned}
$$

where $a$ is a scaling factor such that the thermal strength decays to $20 \%$ of its full strength at mean thermal lifespan $\bar{T}, Q$ is chosen so that it has standard deviation of $4 \mathrm{~m} / \mathrm{s}$ after one mean thermal lifetime $\bar{T}$ to correspond to the largest expected thermal strength. In order to improve estimator performance, $Q$ can be adjusted to better describe thermal dynamics with result from simulation in flight simulator. The estimator is initialized with no priori information on vertical wind speed and maximum uncertainty. The time update step of the $i j^{t h}$ vertical wind estimate is given as

$$
\begin{gathered}
\hat{w}_{z, i j, k \mid k-1}=a \hat{w}_{z, i j, k-1 \mid k-1} \\
P_{i j, k \mid k-1}=a^{2} P_{i j, k-1 \mid k-1}+Q
\end{gathered}
$$

Wind measurement $y_{i j}$ is obtained at aircraft location in $i j^{\text {th }}$ cell, which is corrupted by measurement noise $v_{k}$ with Gaussian distribution $\mathcal{N}(0, R)$, where $R$ has standard deviation of $0.2 \mathrm{~m} / \mathrm{s}$. The measurement update of the $i j^{\text {th }}$ cell is

$$
\begin{gathered}
P_{i j, k \mid k}=\left(P_{i j, k \mid k-1}^{-1}+R^{-1}\right)^{-1} \\
K_{i j, k}=P_{i j, k \mid k} R^{-1} \\
\hat{w}_{z, i j, k \mid k}=\hat{w}_{z, i j, k \mid k-1}+K_{i j, k}\left(y_{i j, k}+\hat{w}_{z, i j, k \mid k-1}\right)
\end{gathered}
$$

In addition to updating the cell where the measurement is taken, a measurement update is performed on the surrounding cells because a thermal radius is typically larger than the width of a cell (and there actually is correlation among cells, although this is not assumed explicitly). However, this update on those cells is done without direct measurements in that cell, and as a result the measurement noise covariance associated with the measurement update on those cells is increased.

\section{B. Aircraft model and low-level control}

It is assumed that the aircraft is equipped with an autopilot that can follow airspeed and turn commands and maintain trimmed flight. An aircraft model representative of the RnR Products SB-XC radio-controlled sailplane is used here (parameters are given in Table 5 in the appendix). First order response to airspeed commands is assumed.

The aircraft state vector $\mathbf{x}$ and control input $\mathbf{u}$ are

$$
\begin{aligned}
& \mathbf{x}=\left[\begin{array}{llllll}
p_{N} & p_{E} & p_{D} & v_{a} & \psi & \gamma
\end{array}\right]^{T} \\
& \mathbf{u}=\left[\begin{array}{ll}
v_{a, c m d} & \dot{\psi}_{c m d}
\end{array}\right]^{T}
\end{aligned}
$$

Aircraft flight path angle is

$$
\gamma=\arctan \frac{C_{D}}{C_{L}}
$$


where the lift coefficient $C_{L}$ is determined from airspeed in trimmed level flight, and $C_{D}$ is a function of $C_{L}$.

The resulting aircraft system equations are

$$
\begin{aligned}
\dot{p}_{N} & =v_{a} \cos \psi \cos \gamma+w_{N} \\
\dot{p}_{E} & =v_{a} \sin \psi \cos \gamma+w_{E} \\
\dot{p}_{D} & =v_{a} \sin \gamma+w_{D} \\
\dot{v}_{a} & =\frac{1}{\tau_{v}}\left(v_{a, c m d}-v_{a}\right) \\
\dot{\psi} & =\dot{\psi}_{c m d} \\
\dot{\gamma} & =\arctan \frac{C_{D}}{C_{L}}-\gamma
\end{aligned}
$$

where $\left[\begin{array}{lll}w_{N} & w_{E} & w_{D}\end{array}\right]^{T}$ is the wind vector at aircraft location. These equations are propagated using fourthorder Runge-Kutta integration with a time step of 0.02 seconds.

Command inputs to low-level controller are derived from the high-level behavior controller described in the next sub-section. A heading controller tracks target waypoint by computing the difference between heading to target waypoint and current heading. For energy exploitation in a thermal, the thermal centering controller presented by Andersson and Kaminer ${ }^{4,5}$ provides required turn rate for a steady turn radius $r$ and airspeed $v_{a}$ given as

$$
\dot{\psi}_{c}=\frac{1}{r} v_{a}-k \ddot{e}_{c v_{a}}
$$

where $k$ is a scale factor to reflect importance of energy "acceleration" $\ddot{e}_{c v_{a}}$. This controller centers a thermal by tuning turn rate so that the rate of change of total energy is maximized.

During exploration the aircraft flies at its best L/D speed (which maximizes range). Assuming a second order polynomial relating sink rate to airspeed (i.e. $v_{a} \sin \gamma=a v_{a}^{2}+b v_{a}+c$ ), the airspeed for best L/D is ${ }^{8}$

$$
v_{L / D}=\sqrt{\frac{c+w_{D}}{a}}
$$

where $w_{D}$ is current vertical wind measurement, $a$ and $c$ are the coefficients of quadratic fit of the SB-XC aircraft sink-rate polar (Table 5 of the Appendix). While thermalling, airspeed to fly is at minimum sink in order to maximize the altitude gain in a thermal. The airspeed at minimum sink is $15.5 \mathrm{~m} / \mathrm{s}$ for the SB-XC aircraft model.

\section{Aircraft flight behaviors}

This section reviews aircraft behaviors including local exploration, global exploration, cruise to thermal, and thermalling mode. Switching between modes occurs at the end of a planning interval $\Delta t_{\text {plan }}$, with mode selection occuring based on aircraft states and thresholds as defined in Table 1. The inclusion of a priori information in mode switching is discussed in Section IV.

Table 1. Summary of behavior switching criteria based on aircraft states and threshold

\begin{tabular}{cc}
\hline \hline Behavior & Criteria \\
\hline Local Exploration & default behavior \\
Global Exploration & mean $\left(\Sigma_{\text {local }}\right) \geq \Sigma_{\text {thresh }}$ \\
Cruise to Thermal & $h<h_{\text {min }}$ \\
Thermalling & $w_{z} \geq v_{\text {MacCready }}(h)$ \\
\hline \hline
\end{tabular}

The objective of exploration is to measure updraft at location with relative high likelihood of thermal formation, and energy map can then be updated. Local exploration explores region bounded by the index $\Delta i_{\text {local }}$ and $\Delta j_{\text {local }}$. Cell associated with first priority is chosen for local exploration. With enough confidence in wind measurement within local small region, global exploration is switched while energy exploitation is not needed. The region bounded by $\Delta i_{\text {global }}$ and $\Delta j_{\text {global }}$ is evenly divided into four quadrants. The center of a quadrant associated with first priority is selected as the target waypoint. 
The energy exploitation mode can be triggered by altitude falling below a threshold or by encountering a strong thermal. The MacCready value of a particular thermal indicates whether it is a "useful" thermal, and it depends on thermal strength and the current aircraft altitude. ${ }^{6,8}$ The MacCready value function developed by Cochrane ${ }^{7}$ is applied to the SB-XC aircraft model: if an updraft greater than current MacCready value is encountered, thermalling mode will be activated immediately. Secondly, if the aircraft altitude falls below an altitude floor $h_{m i n}$, immediate energy exploitation is triggered, and the map of atmospheric energy is used to locate a nearby thermal with adequate strength. The altitude floor corresponds to the level of risk that aircraft is willing to taken. Exploration with lower altitude floor is risky but it provides more time for exploration. With the addition of topography, the importance of $h_{\min }$ (as a height above the maximum terrain elevation) will be investigated in simulation.

\section{Atmospheric Environment}

For soaring flight the key components of an environment are thermal updrafts, topography and solar irradiance. Some assumptions are made to simplify the atmospheric model but it still provides an adequate environment in order to evaluate the guided exploration approach. Final performance validation of the exploration approach is performed in a high fidelity flight simulator with more realistic thermal dynamics and atmospheric environment.

\section{A. Sun incidence}

Solar heating of the ground is a function of the solar incidence angle, with the rate of heat absorption maximized when solar incidence is normal to terrain.

The Solar Position Algorithm (SPA) ${ }^{17}$ developed for solar radiation applications computes azimuth $\theta_{a z}$ and zenith $\theta_{z e}$ angles of the sun given a geographic location and time. The SPA algorithm is accurate with uncertainties of \pm 0.003 degrees from the year -2000 to 6000 .

In the persistent surveillance problem considered here, all aircraft stay within a region covering a few square kilometers. Over this scale the variation in solar azimuth and zenith is small, hence it can be computed once for a single reference latitude and longitude defining the center of the grid. The solar irradiance vector $\hat{\mathbf{r}}_{\text {sun }}$ in east-north-up (ENU) coordinate system can be computed from the azimuth and zenith as

$$
\hat{\mathbf{r}}_{\text {sun }}=\left[\begin{array}{lll}
\sin \theta_{a z} \sin \theta_{z e} & \cos \theta_{a z} \sin \theta_{z e} & \cos \theta_{z e}
\end{array}\right]^{T}
$$

Computing solar incidence angle requires knowledge of the terrain normal vector. The National Elevation Dataset (NED) of the United States Geological Survey (USGS) provides digital elevation data in orthometric height, which is the height above the geoid referenced to the North American Vertical Datum of 1988 (NAVD 88). Over the scale of the grid considered here the variation in geoid height between each grid spacing is small, which has negligible effect on the computation of terrain normal vector. Orthometric height can then be treated as ellipsoidal height. In addition to terrain height, USGS provides terrain cover maps, which would allow more accurate computation of likelihood of thermal trigger with additional information on terrain heat capacity. However, the terrain heat capacity is ignored here to focus on the solar incidence angle.

Elevation data at resolutions of 3 meters, 10 meters and 30 meters are available from USGS. Resolution of 3 meters and 10 meters provides more detail of topography but it significantly increases computational cost. In addition, thermal updrafts have a much larger scale, which ranges from tens of meters to hundreds of meters in core radius. It is reasonable to assume that the variation of elevation in small scale does not affect thermal formation. Therefore, elevation data at resolution of 30 meters provides adequate size for topographic model. Two topographic elevation models of $4 \mathrm{~km}$ by $4 \mathrm{~km}$ are obtained near State College and Warm Springs as shown in Figure 1. The topographic model in State College provides a geographic location with relatively small variation of elevation. The topographic model at Warm Springs provides much larger variation of elevation with ridges that have slope facing in east and west direction.

The terrain normal vector $\hat{\mathbf{n}}_{i j}$ for the $i j^{\text {th }}$ grid cell is computed from the cross product of the two vectors that define diagonals across the cell (Figure 2). It is then normalized so its magnitude is one.

The solar incidence angle $\theta_{\text {incidence }}$ of the $i j^{\text {th }}$ cell can now be computed from the dot product of the unit vector to the sun and the unit vector defining the terrain normal:

$$
\theta_{\text {incidence }, i j}=\arccos \left(\hat{\mathbf{r}}_{\text {sun }} \cdot \hat{\mathbf{n}}_{i j}\right)
$$




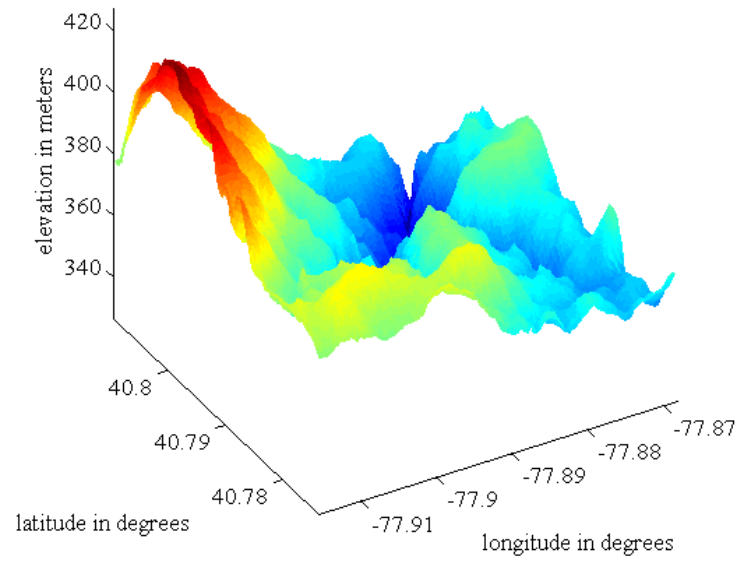

(a) $\mathrm{N} 40.7915^{\circ}$, W $77.8918^{\circ}$ near State College, PA

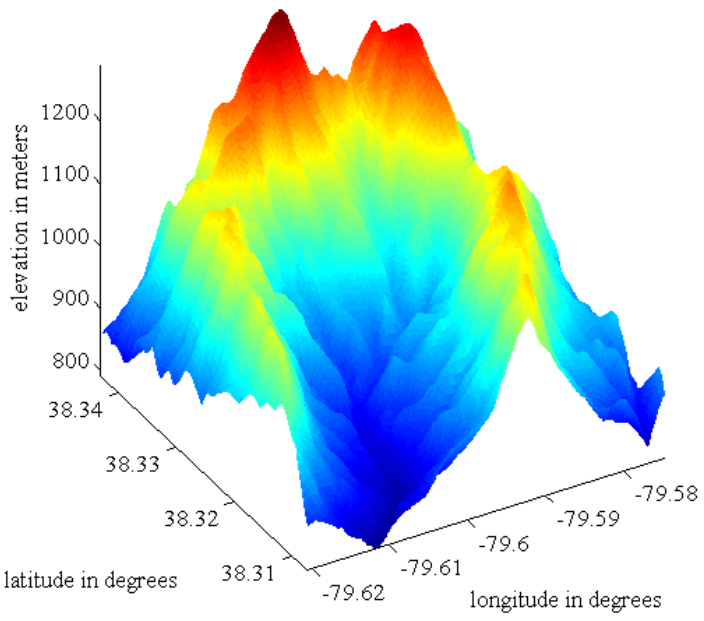

(b) N $38.3251^{\circ}, \mathrm{W} 79.5976^{\circ}$ near Warm Springs, VA

Figure 1. Topographic elevation models used in Monte Carlo simulations

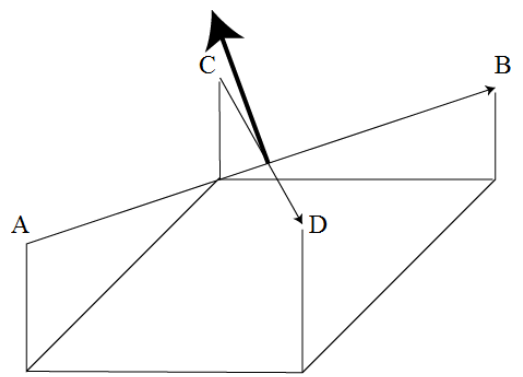

Figure 2. Terrain normal vector defined as vector product of two diagonal vectors

Here $\theta_{\text {incidence }, i j}=0$ means that the sun is overhead and the vector to the sun is exactly perpendicular to terrain, and solar heating will be maximized. $\theta_{\text {incidence }, i j}>\pi / 2$ implies that terrain is shadowed, and solar heating will be zero. A map of solar incidence angle at each grid cell for the region centered on State College, PA is shown in Figure 3.

\section{B. Thermal updraft model}

A thermal updraft model proposed by Gedeon ${ }^{18}$ is used here:

$$
w_{z}\left(x, y, t_{0}\right)=\omega_{0} e^{-\left(\frac{\sqrt{\left(x-x_{0}\right)^{2}+\left(y-y_{0}\right)^{2}}}{R}\right)^{2}}\left[1-\left(\frac{\sqrt{\left(x-x_{0}\right)^{2}+\left(y-y_{0}\right)^{2}}}{R}\right)^{2}\right]
$$

where $\omega_{0}$ is the maximum vertical wind speed, $x_{0}$ and $y_{0}$ are the coordinates of the thermal center, and $R$ is the core radius of thermal. This thermal is shown in Figure 4(a).

Thermals are time-varying, with a typical lifespan of approximately 15 minutes. The time history of a thermal is modeled using sigmoid functions:

$$
\frac{w_{z}(x, y, t)}{w_{z}\left(x, y, t_{0}\right)}=\left(\frac{1}{e^{\eta\left(t-\left(t_{0}+\frac{1}{2} T\right)\right)}+1}+\frac{1}{e^{\eta\left(t-\left(t_{0}-\frac{1}{2} T\right)\right)}+1}-1\right)
$$

where $\eta$ is the scaling factor that determines rise time of full thermal strength. Thus, thermal model can be tuned to more accurately model thermal by changing $\eta$. A time history is shown in Figure 4(b). Typical thermal characteristic over a range of values are used for parameters such as thermal period, thermal radius, 


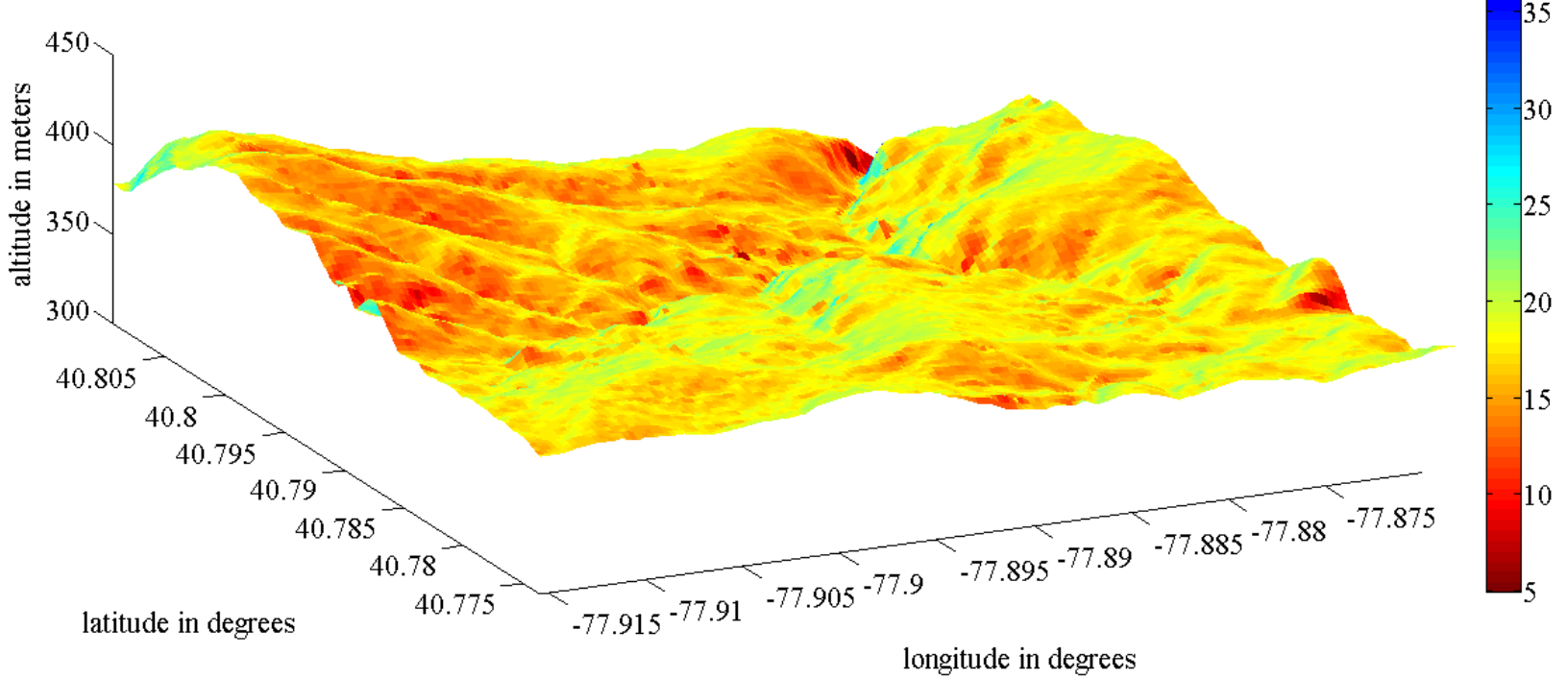

Figure 3. A map of incidence angle in degrees for State College topographic model at noon, on June 6, 2012

\begin{tabular}{cccccc}
\hline \hline parameter & symbol & mean & $\sigma$ & $\max / \mathbf{m i n}$ & units \\
\hline Period & $T_{\text {thermal }}$ & 20 & 7.5 & $60 / 5$ & minutes \\
Radius & $\mathrm{R}$ & 75 & 20 & $200 / 30$ & $\mathrm{~m}$ \\
Core strength & $\omega_{0}$ & -2.56 & 1.5 & $-7 / 1$ & $\mathrm{~m} / \mathrm{s}$ \\
\hline \hline
\end{tabular}

Table 2. Thermal parameters for boundary layer thickness $z_{i}=1500$ meters.

and core strength. It is assumed that these variables have Gaussian distribution, with parameters obtained from Allen ${ }^{19}$ and given in Table 2.

For the Monte Carlo simulation results presented in a subsequent section, thermal generation is partially dependent on incidence angle, with thermals triggering with higher probability where solar incidence is near perpendicular to terrain. The probability of thermal triggering is formulated as a function of solar incidence angle and noise with uniform distribution $\epsilon$ within \pm 0.2 . The noise term introduces randomness to thermal generation:

$$
\mathrm{P}_{\text {thermal }}=\left\{\begin{aligned}
1-\left(\frac{\theta_{\text {incidence }, i j}}{\theta_{\text {limit }}}\right)^{1.5}+\epsilon & \text { if } \theta_{\text {incidence }, i j} \leq \theta_{\text {limit }} \\
0 & \text { if } \theta_{\text {incidence }, i j}>\theta_{\text {limit }}
\end{aligned}\right.
$$

Constraints are imposed on maximum number of thermal in the world, and probability of thermal generated at each time step $\Delta t_{\text {gen }}$ in order to provide more realistic simulation environment so that all thermals are not generated and dissipated at once. The maximum number of thermals $N$ within an area $A$ given by Allen ${ }^{19}$ allows too many thermals for an realistic environment. The maximum number of thermal with a scaling factor $b$ of 3 allows maximum of 20 thermals for a $4 \mathrm{~km}$ by $4 \mathrm{~km}$ map given as

$$
N=\frac{0.6 A}{b z_{i} \bar{R}}
$$

where $z_{i}$ is the boundary layer thickness, and $\bar{R}$ is mean thermal radius. The probability of generating thermal $P_{\text {generate }}$ is inversely proportional to thermal generation interval $\Delta t_{\text {gen }}$ and number of active thermals $n$.

$$
\left.P_{\text {generate }}=\min \left(\frac{\Delta t_{\text {gen }} N}{\bar{T}}\right)(N-n), 1\right)
$$

While the conditions on maximum number of thermal $N$ and probability of generating a new thermal $P_{\text {generate }}$ are satisfied, a new thermal is triggered at a cell associated with the highest $P_{\text {thermal }}$, and its 


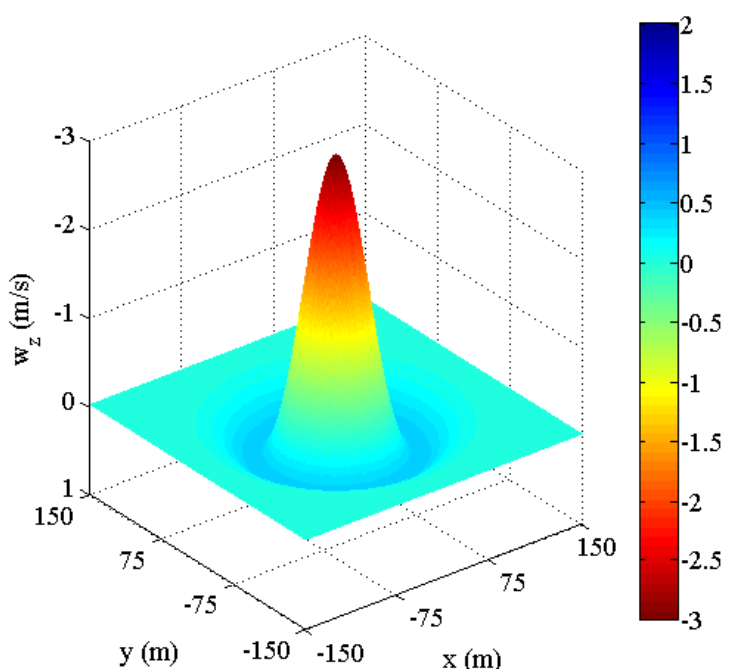

(a) Area of sinking air surrounding core of updraft

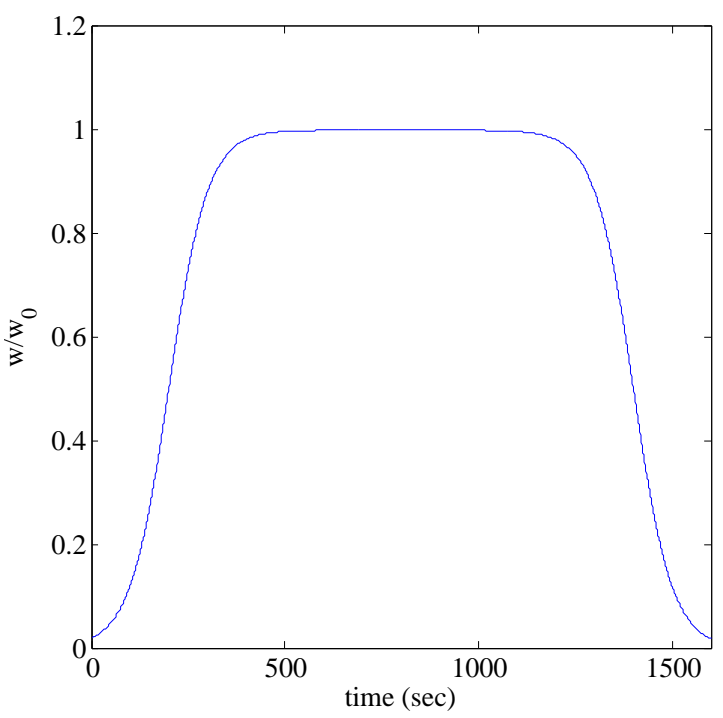

(b) Variation in thermal strength with time

Figure 4. Thermal model

location is subject to the minimum separation distance between thermals, which is approximately four times the core radius of a thermal. Thermal activity is sensitive to the choice of $\theta_{\text {limit }}$, which depends on topographic elevation models.

\section{Exploration Priority}

In earlier work exploration priority was driven purely by uncertainty in the wind map ${ }^{15}$ with regions of high vertical wind speed covariance assigned a high priority for exploration. For large flock sizes (six to eight aircraft) this approach performed very well, ensuring that the environment remained well-explored. However, this approach leads to significant exploration of regions where thermals are unlikely to be found.

One could drive exploration by the likelihood of thermal triggering (here driven by solar incidence). While this will lead to exploration of regions more likely to contain thermals, it is likely to result in "excessive exploration" of these areas, even when no thermals are present there. Further, some terrain maps may have large flat regions where solar incidence is roughly uniform, providing little a priori guidance on search. A hybrid approach that computes an exploration priority based on some function of both vertical wind covariance and solar incidence angle is likely to provide a good solution. In this approach a cell that combines high covariance with near-perpendicular sun incidence will be assigned the highest priority for exploration, thus driving exploration to regions that are both relatively unexplored and likely to contain thermals.

The exploration priority function is formulated by first computing the average value of incidence angle $\bar{\theta}_{\text {incidence }}$ and the standard deviation of the incidence angle $\sigma_{\text {incidence. }}$. The contribution to exploration priority for the $i j^{\text {th }}$ cell due to solar incidence is then defined as

$$
p_{\text {incidence }, i j}=\frac{\bar{\theta}_{\text {incidence }}-\theta_{\text {incidence }, i j}}{\sigma_{\text {incidence }}}
$$

Note that $\theta_{\text {incidence, } i j}$ varies from 0 to $\pi$, with $\theta_{\text {incidence }, i j}=0$ corresponding to solar incidence perpendicular to terrain (leading to highest solar heating). Positive values of $p_{i n c i d e n c e, i j}$ will thus be desirable for exploration.

The net exploration priority $Z_{i j}$ for the $i j^{\text {th }}$ cell is

$$
Z_{i j}=\alpha P_{i j}+(1-\alpha) \operatorname{sgn}\left(p_{\text {incidence }, i j}\right) p_{\text {incidence }, i j}^{2}
$$


The signum function in the second term of the exploration priority equation indicates that cells with higher than average solar incidence angles are less likely to contain thermals, and thus are a lower priority for exploration.

The choice of weight $\alpha$ will have a significant effect on cell exploration priority. Scaling differences in solar incidence angle and vertical wind speed covariance complicate the choice of weight, and Monte Carlo simulations for various flock sizes were conducted to determine a reasonable value of $\alpha$. Results are shown in shown in Figure 5. With an increase of flock size, $\alpha$ of 0.1 results maximum number of thermals found so that it is chosen for exploration priority function.

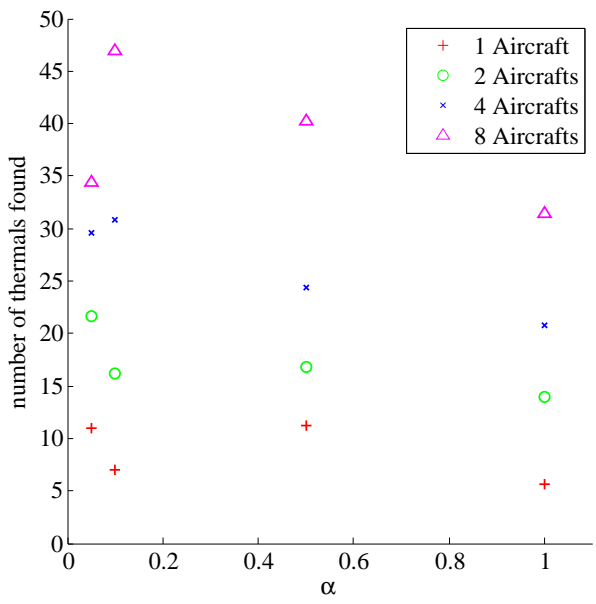

Figure 5. Monte Carlo simulation with flock size of 1, 2, 4 and 8 aircraft and weight factor of of $0.05,0.1,0.5$ and 1 (no priori case) are tested for 5 runs.

\section{Simulation Results}

Simulation results are presented with flock sizes of 1,4 and 8 aircrafts for two topographic models: State College, PA and Warm Springs, VA. The weight factor $\alpha=0.1$ (obtained in the previous section) in exploration priority function is used for all runs. For comparison, a baseline scenario of exploration behavior completely driven by uncertainty in wind estimate is used.

Three different altitude floors (triggering immediate mode switch to energy exploitation) are used: $h_{\text {min }}=$ $200 \mathrm{~m}, 500 \mathrm{~m}$, and $800 \mathrm{~m}$ above the maximum terrain elevation in each topographic model. The additional altitude of 200 meters above maximum terrain elevation provides the aircraft just enough height to traverse the map at best L/D speed. Thermal activity is expected to become weaker at sunset so that simulation starts at 11 a.m. on June 6, 2012 with duration of six hours. Thermals are not generated at locations with an incidence angle $\theta_{\text {limit }}$ is greater than $\frac{\pi}{4}$. Touch down is defined when the altitude of an aircraft is below the elevation in topographic model. Monte Carlo simulations for each case uses the same set of random number sequence in order to provide the identical atmospheric environment. Monte Carlo simulation of 100 runs use different seeds of random number, which generate different thermal activity for each run. Aircraft are initialized at the center of the map with different headings with an initial altitude of 1500 meters above the maximum terrain height. Aircraft starts at the ceiling altitude of 2000 meters and 2800 meters in State College and Warm Springs respectively. The height of convective boundary layer is often higher than those starting altitude. Communication between aircrafts is available for all time to share wind measurement at the rate of 3 seconds.

Monte Carlo simulation results are shown as boxplots in Figures 6, 7 and 8. For each boxplot, central line is the median; edges of the box is defined by the interquartile range (IQR); whiskers extend datapoints from $q 1-1.5(q 1-q 3)$ to $q 3+1.5(q 1-q 3)$; outliers "+" are plotted individually. Boxplots of flight endurance with various flock size, topographic model, and altitude floor are provided in the following.

Results summarizing the Monte Carlo simulations for a single aircraft are shown in Figure 6. For the State College elevation model, the guided exploration approach improves endurance at all levels of altitude floor with an increase of median and IQR as shown in Figure 6(a). There are only 4 outliers out of 100 runs 


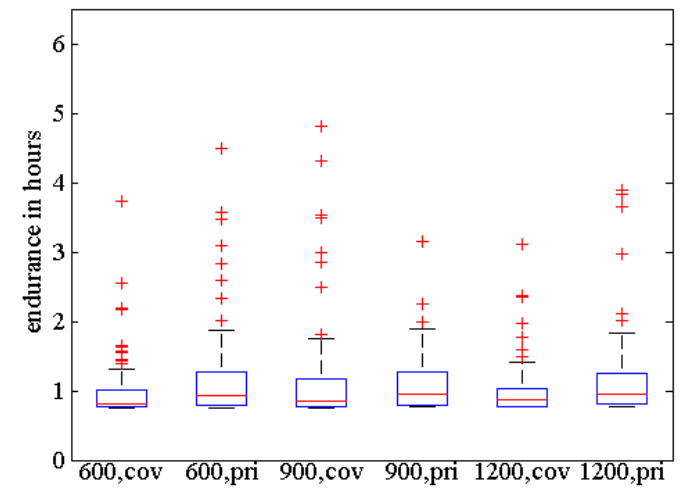

(a) State College, PA

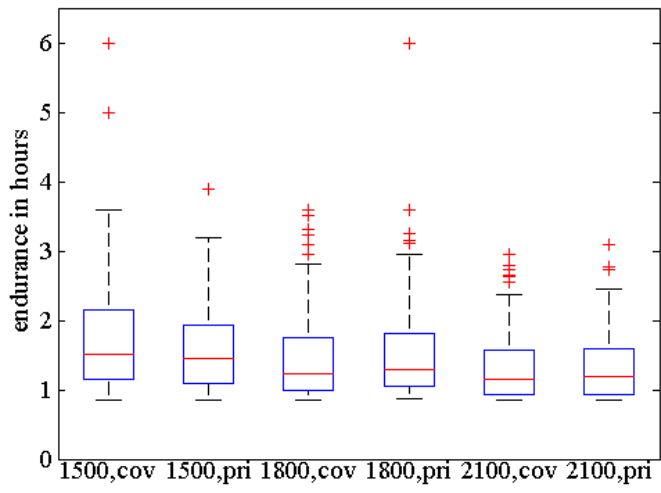

(b) Warm Springs, VA

Figure 6. Endurance for 1 aircraft using covariance and exploration priority for exploration

at altitude floor of 900 meters that guided exploration is outperformed. However, there is no performance difference with guided exploration while flying at high altitude or flying more aggressively at lower altitude. For the Warm Springs elevation model in Figure 6(b), guided exploration gives slightly lower endurance at the altitude floor of 1500 meters. Exploration priority gives slightly better performance with an increase of altitude floor. An decrease trend of endurance over range of altitude floors indicates that single aircraft has to fly more aggressively to look for thermals. Overall, single aircraft can still benefit from priori information with two distinguish topographic models but persistent flight of 6 hours is not achievable with single aircraft. Note that this is dependent on the atmospheric parameters used in simulations: flight experience by other researchers (Edwards, ${ }^{3}$ Allen, ${ }^{2}$ and Andersson ${ }^{5}$ ) suggests that in practice significantly longer flight times are achievable.

With an increase of flock size of 4 aircrafts, guided exploration for State College terrain provides significant performance gain at altitude floor of 600 meters as shown in $7(\mathrm{a})$. The performance gain from priori at higher altitude floor is relatively negligible. Among the three levels of altitude floor, flying at lower altitude floor with priori provides slightly better performance but performance gain starts to decrease; the IQR does not always increase with lower altitude floor. For Warm Springs terrain, Figure 7(b) shows similar result as the single aircraft case. An increase of altitude floor results performance gain from priori eventually. Again, there is still a decreasing trend in endurance with an increase in altitude floor is shown for both topographic models, which indicates flying at low altitude at more risk is more preferable with a flock size smaller than 4 aircrafts.

For the 8 aircrafts case, guided exploration provides significant performance gain for State College terrain as shown in 8(a). An relative small improvement occurs at altitude floor of 600 meters. It is important to denote that the decrease trend of endurance with increase of altitude floor in Figure 6 and Figure 7 is absence here. There is no benefit from flying more aggressively at low altitude. The advantage of flying low is compensated by the larger flock size. It becomes unnecessary to take more risk, and flying at high altitude still provide enough time in exploration mode to identify thermal. For the Warm Springs terrain in Figure 8(b), once again, guided exploration is outperformed at altitude floor of 1500 meters until the next level of altitude floor. Result from Warm Springs terrain also confirms that it becomes unnecessary to fly at lower altitude to yield more exploration time. Both topographic models indicates that with a large enough flock size, flying at higher risk is unnecessary.

Overall, guided exploration in State College terrain indicates performance gain for all cases. However, it is not the case for the Warm Springs terrain. Guided exploration with different flock sizes is always outperformed at the altitude floor of 1500 meters, and performance gain occurs at higher altitude floor. It is because there is a large area associated with high likelihood of thermal formation throughout the entire simulation. The instance of a incidence angle map of two terrains is shown in Figure 9 for comparison. The area associated with high likelihood of thermal formation in Warm Springs terrain is significantly larger. This simulation result confirms that guided exploration with higher altitude floor is more preferable for high 


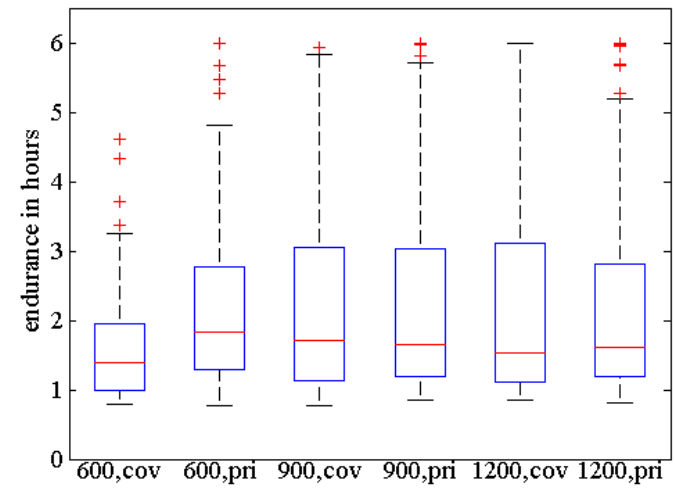

(a) State College, PA

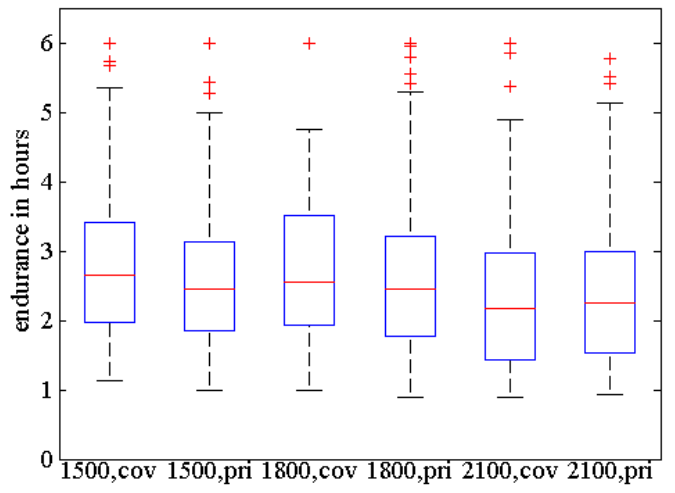

(b) Warm Springs, VA

Figure 7. Endurance for a flock of 4 aircrafts using covariance and exploration priority for exploration

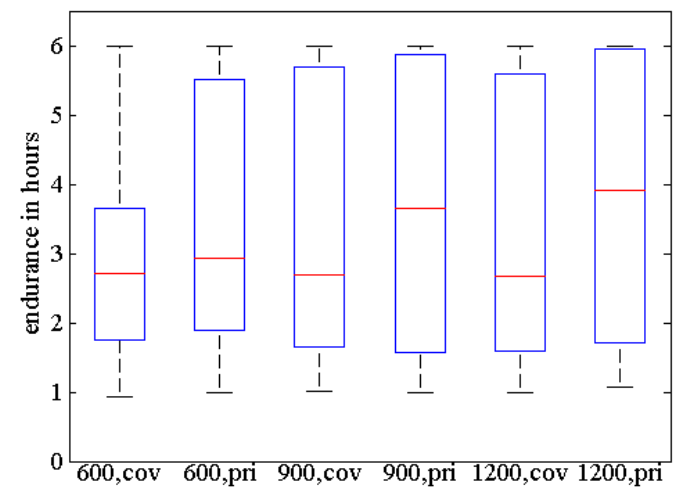

(a) State College, PA

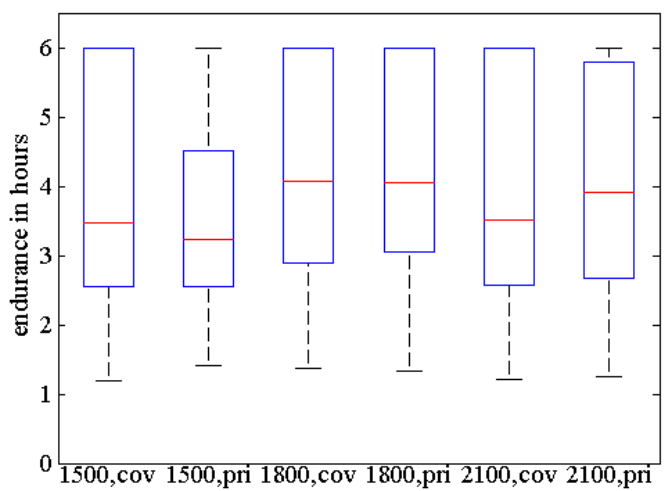

(b) Warm Springs, VA

Figure 8. Endurance for a flock of 8 aircrafts using covariance and exploration priority for exploration

uncertainty in thermal distribution regardless the flock size. However, some further tuning of the weight $\alpha$ may improve performance across all flock sizes.

Simulation result from State College terrain shows an overall improvement in endurance as summarized in Table 3 except the single aircraft case with altitude of 900 meters due to outliers. With the same flock size, guided exploration does not always gives the largest median of endurance at the lowest altitude floor. However, maximum percentage gain occurs at the lowest altitude floor for all flock sizes. It makes sense because lower altitude floor gives more time to be spent in exploration mode. Guided exploration results significant performance gain, which can be easily seen from the number of successful runs survived $90 \%$ of simulation time. On the other hand, result from Warm Springs terrain as shown in Table 4 indicates that guided exploration does not give much improvement over the covariance approach. The highest performance loss occurs at the lowest altitude floor because of the large area associated with high likelihood of thermal formation. With the increase of flock size and attitude floor to 8 aircrafts and 2100 meters respectively, the difference in number of runs survived $90 \%$ of simulation time between covariance and priori driven approaches becomes smaller. It further confirms that higher altitude floor should be set for guided exploration with unknown thermal distribution. The covariance approach has similar performance for the two terrains which make sense because it should be independent of terrain. Therefore, improvement in endurance by guided exploration with a flock size of 8 aircrafts and operational altitude of 1100 meters is achievable even with 


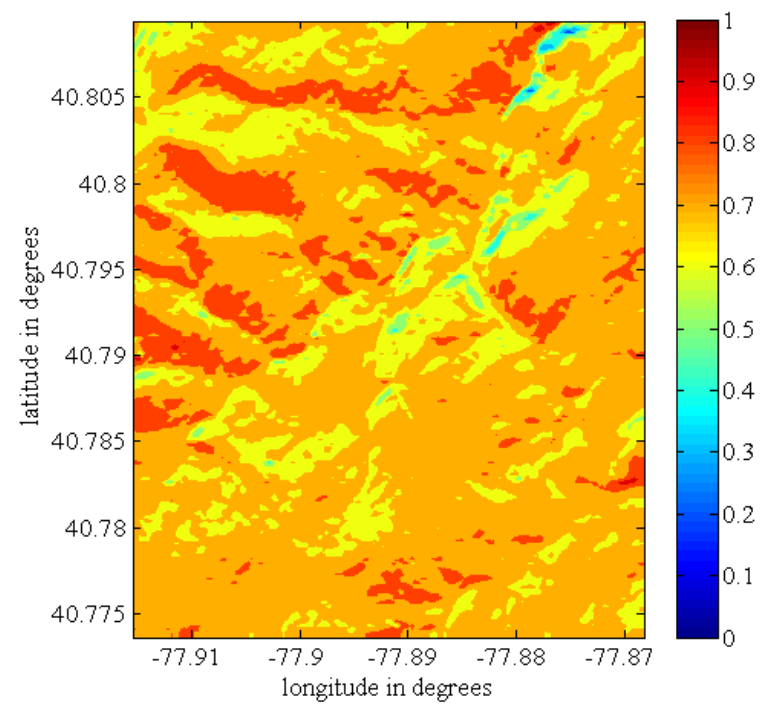

(a) $4 \mathrm{~km} \mathrm{x} 4 \mathrm{~km}$ region in State College, PA

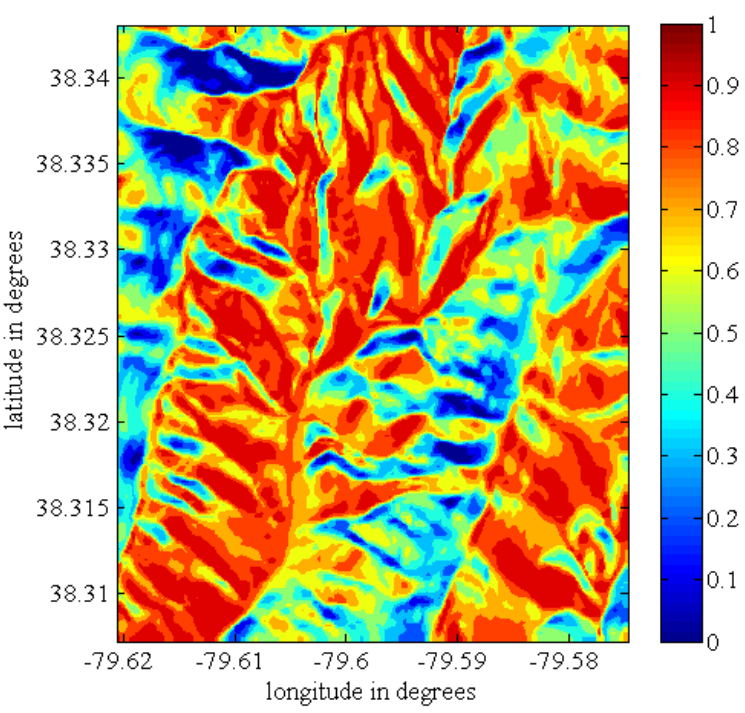

(b) $4 \mathrm{~km} \mathrm{x} 4 \mathrm{~km}$ region in Warm Springs, VA

Figure 9. Contour plots of thermal trigger probability based on incidence angle at noon on June 6, 2012. Region in red represents high likelihood of thermal formation.

high uncertainty in thermal distribution.

Table 3. A summary of Monte Carlo result for State College terrain including outliers.

\begin{tabular}{ccccccc}
\hline \hline \# aircraft & $h_{\text {min }}$ & $t_{\text {mean,cov }}$ & $t_{\text {mean,priori }}$ & $\%$ gain $t_{\text {mean }}$ & \# run $t_{90 \%, \text { cov }}$ & \# run $t_{90 \%, \text { priori }}$ \\
\hline 1 & $600 \mathrm{~m}$ & $.99 \mathrm{~h}$ & $1.19 \mathrm{~h}$ & 20.2 & 0 & 0 \\
1 & $900 \mathrm{~m}$ & $1.14 \mathrm{~h}$ & $1.09 \mathrm{~h}$ & -4.4 & 0 & 0 \\
1 & $1200 \mathrm{~m}$ & $.99 \mathrm{~h}$ & $1.15 \mathrm{~h}$ & 16.2 & 0 & 0 \\
4 & $600 \mathrm{~m}$ & $1.63 \mathrm{~h}$ & $2.19 \mathrm{~h}$ & 34.4 & 0 & 4 \\
4 & $900 \mathrm{~m}$ & $2.21 \mathrm{~h}$ & $2.30 \mathrm{~h}$ & 4.1 & 6 & 8 \\
4 & $1200 \mathrm{~m}$ & $2.23 \mathrm{~h}$ & $2.30 \mathrm{~h}$ & 3.1 & 9 & 27 \\
8 & $600 \mathrm{~m}$ & $2.85 \mathrm{~h}$ & $3.39 \mathrm{~h}$ & 18.9 & 6 & 38 \\
8 & $900 \mathrm{~m}$ & $3.31 \mathrm{~h}$ & $3.70 \mathrm{~h}$ & 11.8 & 29 & 41 \\
8 & $1200 \mathrm{~m}$ & $3.39 \mathrm{~h}$ & $3.83 \mathrm{~h}$ & 13.0 & 28 & 9 \\
\hline \hline
\end{tabular}

\section{Coordinated Thermal Mapping in High Fidelity Flight Simulator}

Silent Wings is a commercially available multi-player soaring simulator (http://www.silentwings.no/). It contains a dynamic thermal model, orographic lift, wind shear, and is capable of correctly modeling extreme flight maneuvers such as spins.

Simulation results from single aircraft and two aircrafts are provided. A schematic of the simulation setup for two aircrafts is shown in Figure 10. A linear aerodynamics model of a SB-XC motor glider is used. The $4 \mathrm{~km} \mathrm{x} 4 \mathrm{~km}$ topographic elevation model is generated in Silent Wings as a scenery, which is centered at $\mathrm{N} 37.1972^{\circ}$, W 80.5814 (near Blacksburg, VA).

Low-level controller is implemented on an Arduino Mega 2560, and serves as an autopilot to track heading and airspeed commands (Section B). The autopilot receives state information from Silent Wings and sends commands to Silent Wings via UDP, and it communicates with the higher level controllers and mapping algorithm via a serial port. 
Table 4. A summary of Monte Carlo result for Warm Springs terrain including outliers.

\begin{tabular}{ccccccc}
\hline \hline \# aircraft & $h_{\text {min }}$ & $t_{\text {mean,cov }}$ & $t_{\text {mean }, \text { priori }}$ & \% gain $t_{\text {mean }}$ & \# run $t_{90 \%, \text { cov }}$ & \# run $t_{90 \%, \text { priori }}$ \\
\hline 1 & $1500 \mathrm{~m}$ & $1.76 \mathrm{~h}$ & $1.58 \mathrm{~h}$ & -10.2 & 1 & 0 \\
1 & $1800 \mathrm{~m}$ & $1.47 \mathrm{~h}$ & $1.56 \mathrm{~h}$ & 6.1 & 0 & 1 \\
1 & $2100 \mathrm{~m}$ & $1.33 \mathrm{~h}$ & $1.34 \mathrm{~h}$ & 0.8 & 0 & 0 \\
4 & $1500 \mathrm{~m}$ & $2.84 \mathrm{~h}$ & $2.67 \mathrm{~h}$ & -6.0 & 5 & 3 \\
4 & $1800 \mathrm{~m}$ & $2.82 \mathrm{~h}$ & $2.67 \mathrm{~h}$ & -5.3 & 5 & 6 \\
4 & $2100 \mathrm{~m}$ & $2.36 \mathrm{~h}$ & $2.38 \mathrm{~h}$ & 0.8 & 3 & 3 \\
8 & $1500 \mathrm{~m}$ & $3.95 \mathrm{~h}$ & $3.58 \mathrm{~h}$ & -9.4 & 29 & 30 \\
8 & $1800 \mathrm{~m}$ & $4.23 \mathrm{~h}$ & $4.22 \mathrm{~h}$ & -0.2 & 40 & 30 \\
8 & $2100 \mathrm{~m}$ & $3.98 \mathrm{~h}$ & $4.07 \mathrm{~h}$ & 2.3 & 33 & \\
\hline \hline
\end{tabular}

The priori information (Section III), mapping algorithm (Section A), behavior determination controller (Section C), and exploration priority function (Section IV) are implemented in Simulink as C-Mex S-function blocks, and run on a laptop computer. The distribution of updraft measurement among aircrafts is performed via UDP at the rate of 3 seconds.

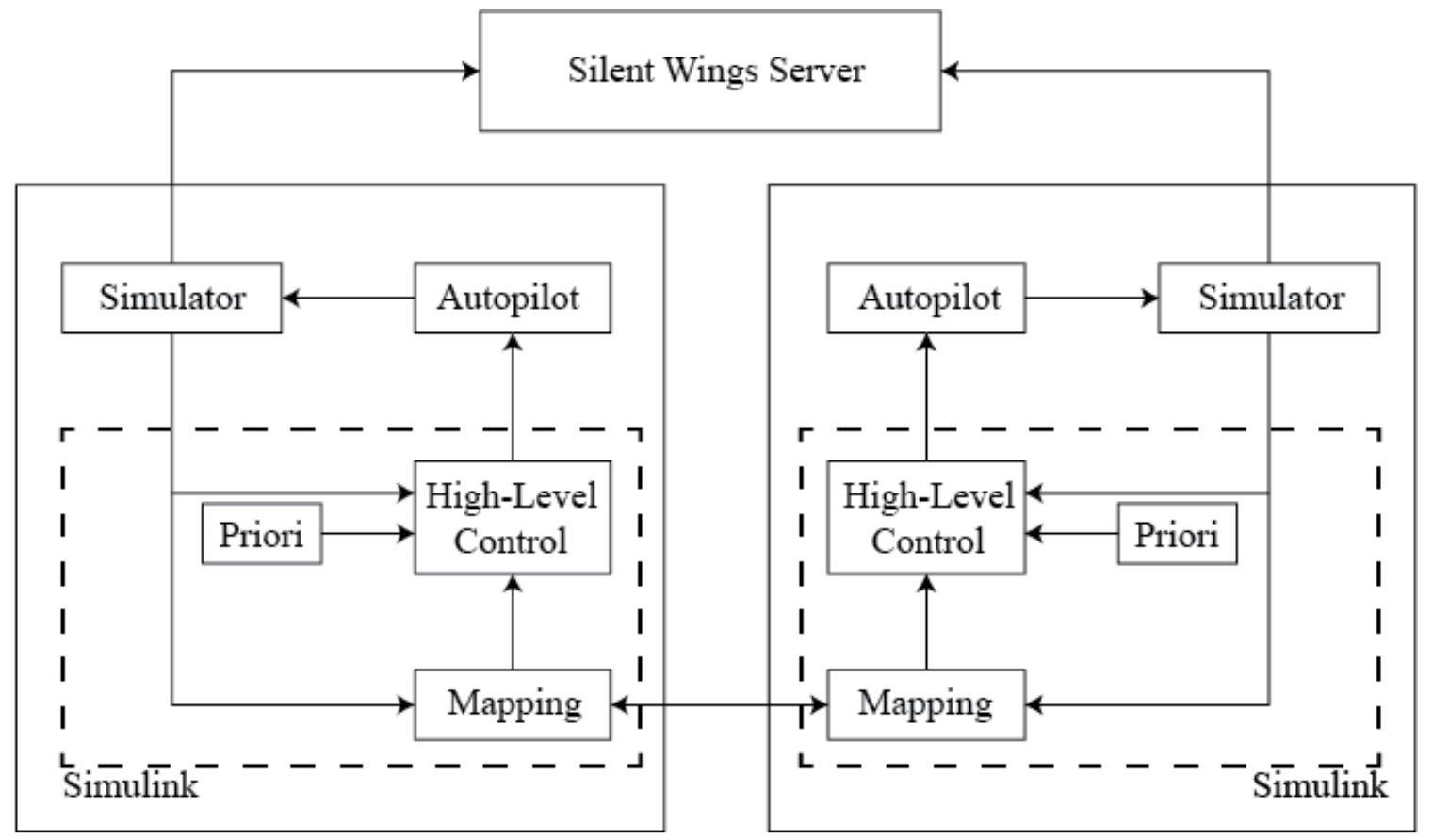

Figure 10. A system overview of Silent Wings simulation setup for two aircrafts

Result from the single aircraft case is shown in Figure 11. The flight path in Figure 11(a) and Figure 11(c) indicate a more favorable regions on the lower left and upper right of the map associated with high likelihood of thermal formation. In fact, the actual thermals are generated nearby these region and no thermal generated in the middle of the map as shown in Figure 12. Exploration in the middle of the map is effectively avoided. For both runs, improvement in endurance is achieved by harvesting significant amount of energy available in thermals. Since the strength of thermal is stronger than the minimum thermal strength computed from MacCready function, exploitation mode is switched while the altitude of aircraft is still above the altitude floor of 800 meters. For the second case, the aggressive exploration approach allows the aircraft to locate the thermal at the threshold altitude. While the thermal strength is smaller than the MacCready value, the aircraft exits the thermals and switches back to exploration mode. A visualization of the behavior switching is shown in Figure 13. 


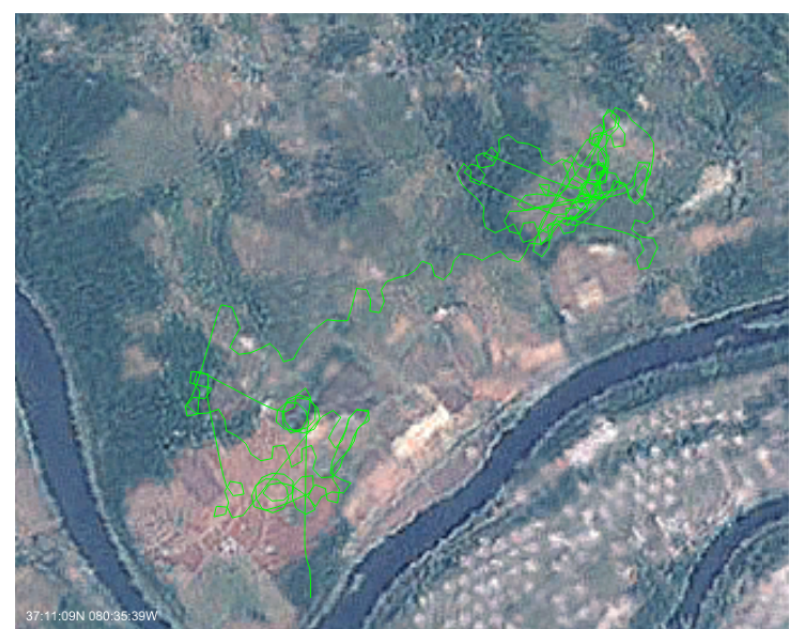

(a) History of flight path

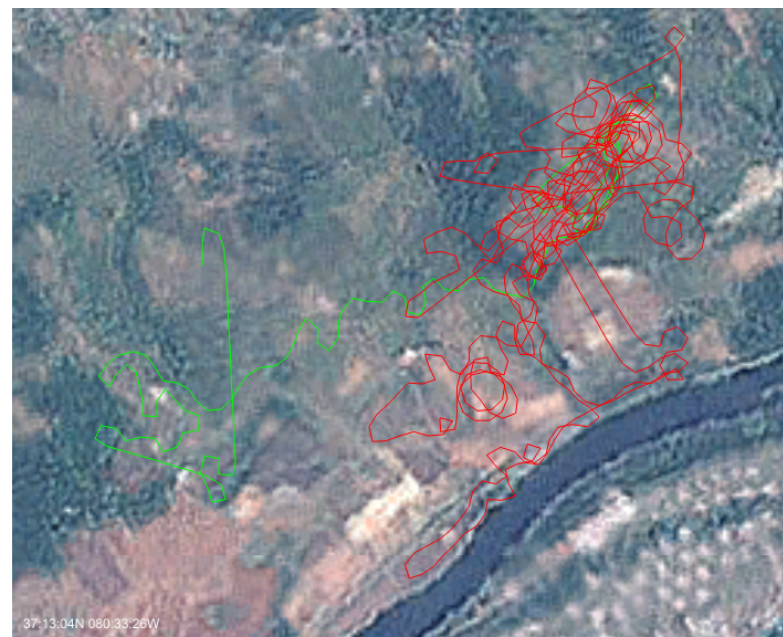

(c) History of flight path

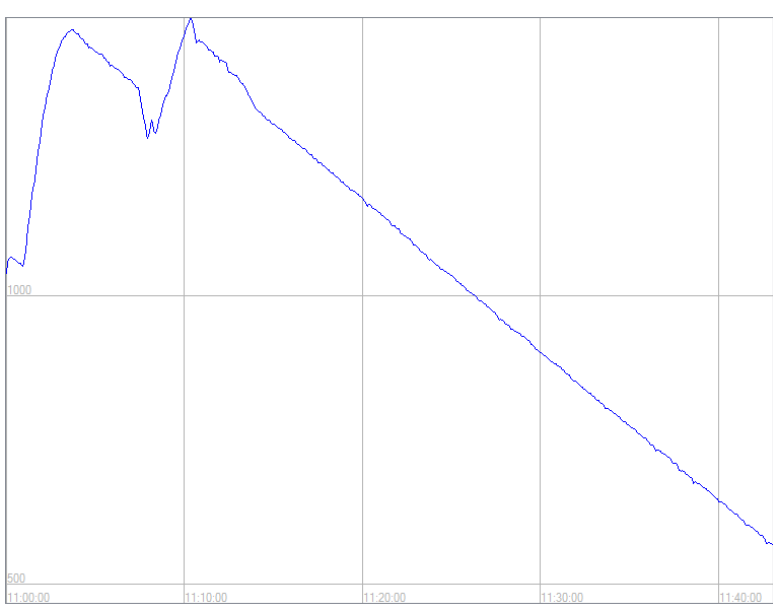

(b) Starting altitude at 1000 meters

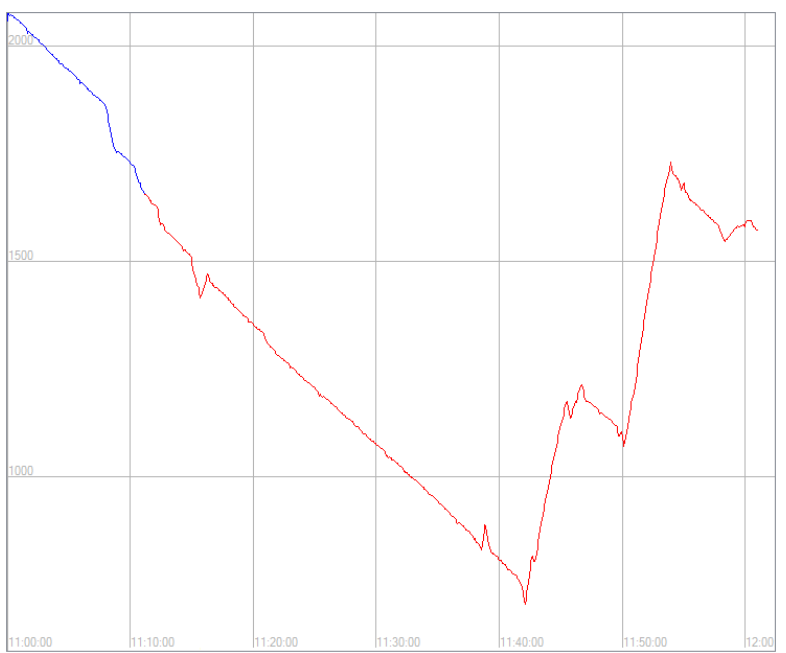

(d) Starting altitude at 2000 meters

Figure 11. Simulations of single aircraft case with thermals setting at medium activity, strength, and size. Altitude floor $h_{\min }$ is $\mathbf{2 0 0}$ meters above maximum height of the terrain. Since the thermal cheat is turned on for visualization, the portion of flight path (c) and altitude plot (d) is shown in red.

Simulation result from coordinated autonomous soaring is shown in Figure 14. Two aircraft are initialized at the center of the map with the same simulation setting as the single aircraft case. Initially, aircraft perform local exploration for nearby cells until the confidence in measurement is above the threshold level. The aircrafts spread out to explore regions with first priority as shown in Figure 14(a) and Figure 14(b). While immediate energy gain is required, the aircraft searches for updrafts within reachable distance at current altitude. If energy gain is available within the glide range, location of updraft is selected as target waypoint. The use of energy map allows updraft to be shared with aircraft at distance away as shown in Figure 14(c) and Figure 14(d). While the bottom aircraft locates a thermal, the location and thermal strength is available to the top aircraft for energy exploitation. If updraft is not available, aircraft continues to perform exploration. The effectiveness of guided exploration can be seen by comparing the actual thermal location and history of flight path as shown in the Figure 12(b) and 14(c). Aircraft only target location with actual thermal for exploration while the region without thermal is avoided. 


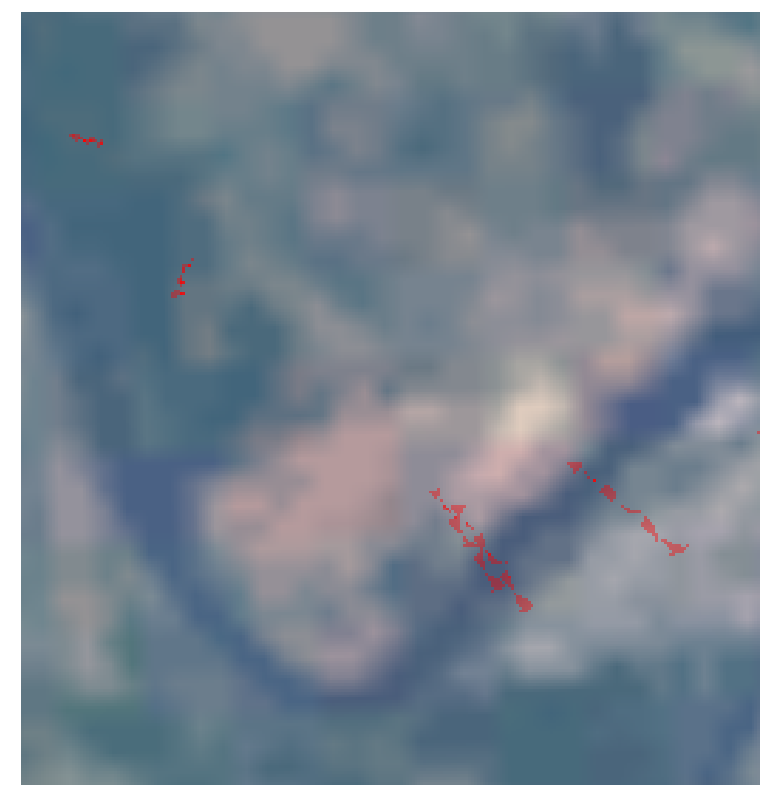

(a) Actual thermal activity at 11 a.m.

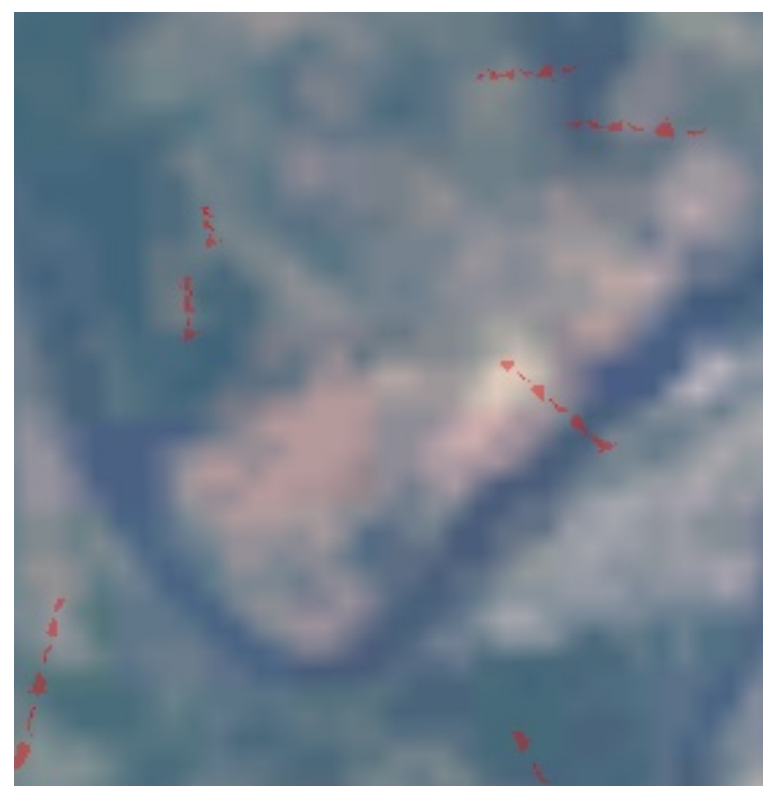

(b) Actual thermal activity at 11:30 a.m.

Figure 12. Distribution of thermal on June 6, 2012. The location of thermals varies slightly for each simulation run. But these locations still indicate a high likelihood of thermal in general.

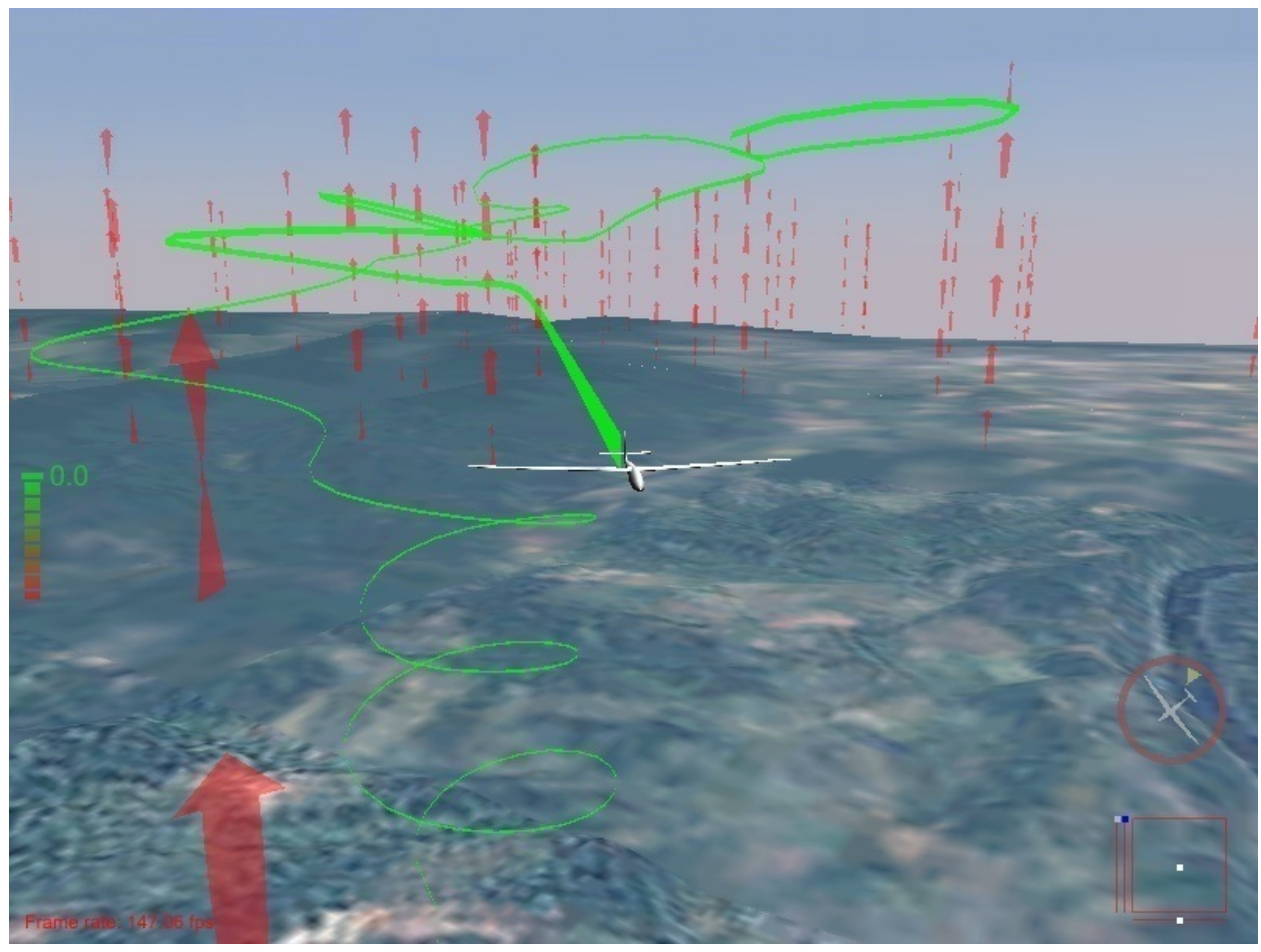

Figure 13. Demonstration of behavior switching for autonomous soaring flight. The aircraft switches to thermalling mode to exploit energy in a thermal. Local exploration mode is switched while enough energy is acquired.

\section{Conclusion}

This research has extended earlier research on coordinated mapping and exploration. An exploration priority function is developed by the combination of covariance in wind estimation, topographic elevation 


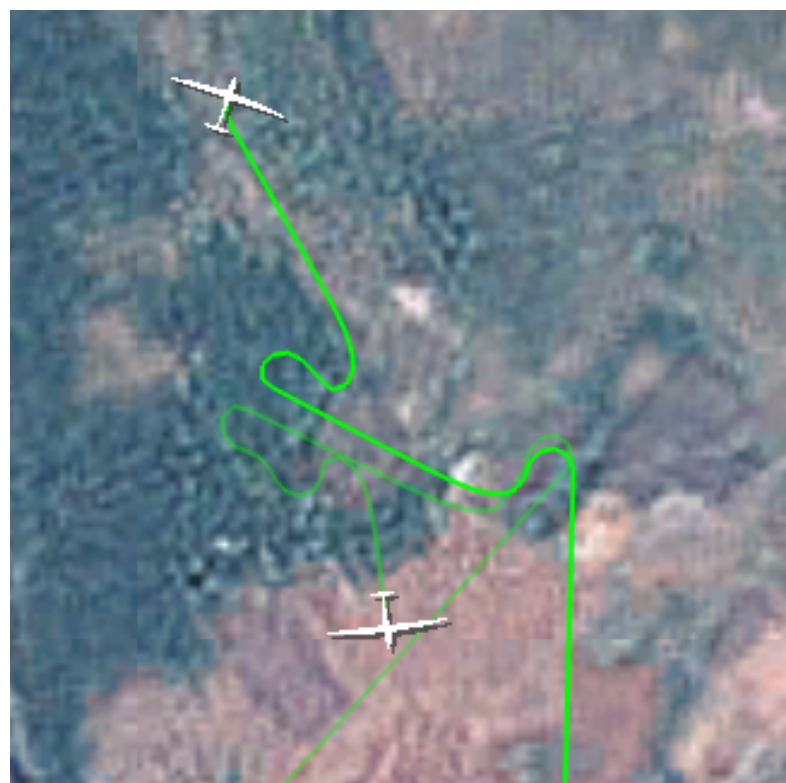

(a) Switch from local to global exploration

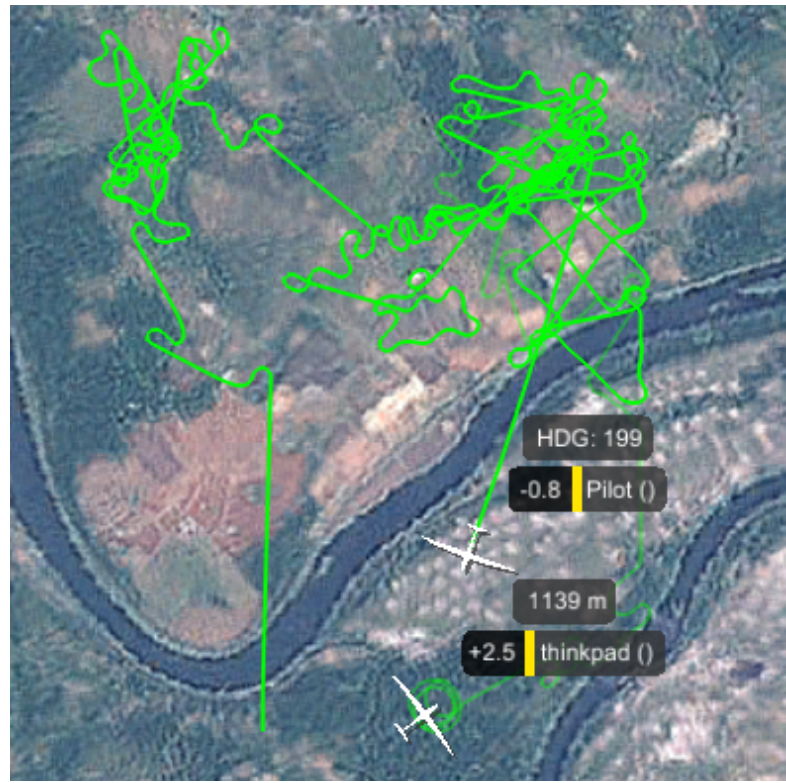

(c) Share the location of thermal updraft

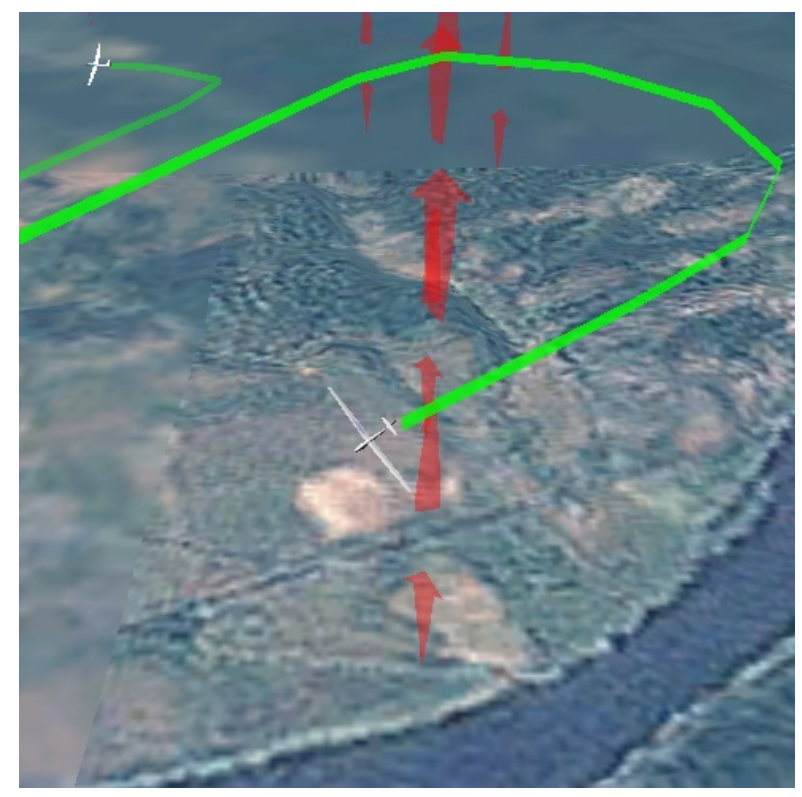

(b) Spread out with confidence in wind estimate

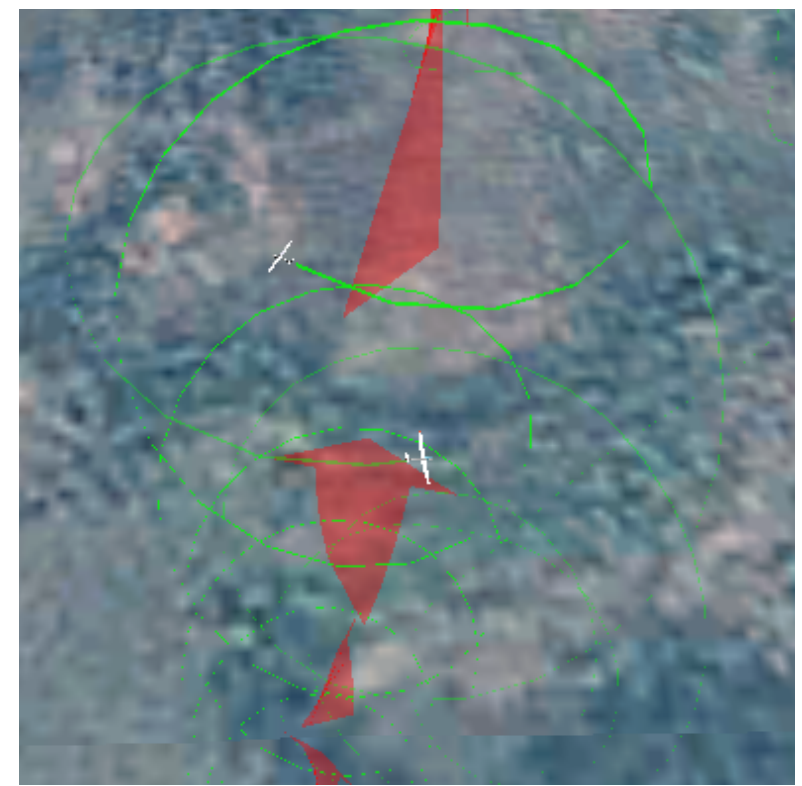

(d) Energy exploitation in a thermal

Figure 14. Demonstration of coordinated mapping and guided exploration for autonomous soaring flight.

data and solar irradiance. A flock of aircraft explores regions associated with both a high likelihood of thermal trigger and a high uncertainty in vertical wind estimates.

The presence of thermal updrafts in the world is well mapped. A trade off between flock size and altitude floor was investigated. A single aircraft requires more time in exploration mode in order to take more measurements. However, the performance gain from flying at lower altitude diminishes with increasing flock size. Eventually, a low altitude floor results in a performance loss for 8 aircraft because large flock size provides enough resources in exploration mode to locate available thermals. In addition, the effectiveness of the incidence angle map on guided exploration was evaluated. Significant improvement can be obtained while the incidence angle map gives clear indication of locations with high likelihood of thermal trigger. However, if the incidence angle map contains a large area with uniform likelihood of thermal trigger, a priori 
information is not very useful and the guided exploration approach can be outperformed. Additional tuning of the relative weight between a priori information and the thermal map may improve performance, as would incorporating addition information such as terrain albedo. Using a higher altitude floor, guided exploration is able to catch up with covariance approach. Therefore, the confidence in thermal prediction determines the level of risk that can be taken. A operational altitude of 1100 meters should be set for an unknown thermal distribution. Lastly, since all simulation result are generated with gliding flight, additional endurance can be expected by powering the aircraft to gain altitude before touch down. Long duration flight of six hours with coordinated autonomous soaring is achievable with small UAVs by incorporating priori information.

Autonomous soaring flight in the Silent Wings simulator demonstrates the feasibility of coordinated soaring flight in a more realistic atmospheric environment. The exploration priority function avoids a large area where the likelihood of thermal formation is small. The actual number of thermal is ranging from 4 to 7 with medium thermal setting. Both simulation cases are still able to locate thermal with the use of exploration priority function.

\section{Acknowledgments}

This research was funded by the Office of Naval Research under Grant N000141110656. The Arduino autopilot (The Raptor) was developed by Nathan Depenbusch and Shawn Daugherty from the Air Vehicle Intelligence and Autonomy Laboratory (AVIA).

\section{References}

\footnotetext{
${ }^{1}$ Allen, M. J., "Autonomous Soaring for Improved Endurance of a Small Uninhabited Air Vehicle," 43rd AIAA Aerospace Sciences Meeting and Exhibit, American Institute of Aeronautics and Astronautics, Reno, Nevada, January 2005.

${ }^{2}$ Allen, M. J. and Lin, V., "Guidance and Control of an Autonomous Soaring Vehicle with Flight Test Results," AIAA Aerospace Sciences Meeting and Exhibit, AIAA Paper 2007-867, American Institute of Aeronautics and Astronautics, Reno, Nevada, January 2007.

${ }^{3}$ Edwards, D. J., "Implementation Details and Flight Test Results of an Autonomous Soaring Controller," AIAA Guidance, Navigation and Control Conference, American Institute of Aeronautics and Astronautics, Reston, Virginia, August 2008.

${ }^{4}$ Anderson, K. and Kaminer, I., "On Stability of a Thermal Centering Controller," AIAA Guidance, Navigation, and Control Conference, Chicago, Illinois, August 10-13 2009.

${ }^{5}$ Anderson, K., Kaminer, I., and Jones, K. D., "Autonomous Soaring; Flight Test Results of a Thermal Centering Controller," AIAA Guidance, Navigation and Control Conference, AIAA Paper 2010-8034, American Institute of Aeronautics and Astronautics, Toronto, Canada, August 2010.

${ }^{6}$ MacCready Jr., P. B., "Optimum Airspeed Selector," Soaring, January-February 1958, pp. 10-11.

${ }^{7}$ Cochrane, J. H., "MacCready Theory with Uncertain Lift and Limited Altitude," Technical Soaring, Vol. 23, No. 3, July 1999, pp. 88-96.

${ }^{8}$ Reichmann, H., Cross-Country Soaring, Thomson Publications, Santa Monica, California, 1978.

${ }^{9}$ Arho, R., "Optimal Dolphin Soaring as a Variational Problem," OSTIV Publication XIII, Organisation Scientifique et Technique Internationale du Vol à Voile, 1974

${ }^{10}$ Metzger, D. E. and Hedrick, J. K., "Optimal Flight Paths for Soaring Flight," Journal of Aircraft, Vol. 12, No. 11, 1975, pp. 867-871.

${ }^{11}$ Sandauer, J., "Some Problems of the Dolphin-Mode Flight Technique," OSTIV Publication XV, Organisation Scientifique et Technique Internationale du Vol à Voile, 1978.

${ }^{12}$ de Jong, J. L., "The Convex Combination Approach: A Geometric Approach to the Optimization of Sailplane Trajectories," OSTIV Publication XVI, Organisation Scientifique et Technique Internationale du Vol à Voile, 1981, pp. $182-201$.

${ }^{13}$ Pierson, B. L. and Chen, I., "Minimum Altitude Loss Soaring in a Specified Vertical Wind Distribution," NASA Conference Publication 2085, Science and Technology of Low Speed and Motorless Flight, edited by P. W. Hanson, NASA, Hampton, Virginia, March 1979, pp. 305-318.

${ }^{14}$ Sander, G. and Litt, F. X., "On Global Optimal Sailplane Flight Strategy," NASA Conference Publication 2085, Science and Technology of Low Speed and Motorless Flight, edited by P. W. Hanson, NASA, Hampton, Virginia, March 1979, pp. $355-376$.

${ }^{15}$ Depenbusch, N. T. and Jack W, L., "Coordinated Mapping and Exploration for Autonomous Soaring," Infotech@Aerospace 2011, AIAA Paper 2011-1436, American Institute of Aeronautics and Astronautics, St. Louis, Missouri, 2011.

${ }^{16}$ Chakrabarty, A. and Langelaan, J. W., "Energy-based Long-range Path Planning for Soaring-capable UAVs," Journal of Guidance, Control and Dynamics, Vol. 34, No. 4, 2011, pp. 1002-1015.

${ }^{17}$ Reda, I. and Andreas, A., "Solar Position Algorithm for Solar Radiation Applications," Tech. rep., National Renewable Energy Laboratory, Golden, Colorado, 2004.

${ }^{18}$ Gedeon, J., "Dynamic Analysis of Dolphin Style Thermal Cross Country Flight," Proceedings of the XIV OSTIV Congress, Organisation Scientifique et Technique Internationale du Vol à Voile, 1974.
} 
${ }^{19}$ Allen, M. J., "Updraft Model for Development of Autonomous Soaring Uninhabited Air Vehicles," 44th AIAA Aerosciences Meeting, AIAA Paper 2006-1510, American Institute of Aeronautics and Astronautics, January 2006.

\section{Appendix: Vehicle Properties}

Simulation results are based on the $\mathrm{RnR}$ products SB-XC radio control glider.

Note that a fourth order polynomial is used to relate $C_{D}$ to $C_{L}$ : this provided a better fit to the computed data over the full speed range.

Table 5. Parameters for SB-XC glider.

\begin{tabular}{ccc}
\hline \hline variable & value & description \\
\hline $\mathrm{m}$ & $10 \mathrm{~kg}$ & mass \\
$\mathrm{S}$ & $1 \mathrm{~m}^{2}$ & wing area \\
$f\left(C_{L}\right)$ & $0.1723 C_{L}^{4}-0.3161 C_{L}^{3}+0.2397 C_{L}^{2}$ & \\
& $-0.0624 C_{L}+0.0194$ & \\
$v_{a, \text { min }}$ & $12 \mathrm{~m} / \mathrm{s}$ & \\
$v_{a, \text { max }}$ & $35 \mathrm{~m} / \mathrm{s}$ & efficiency of the propeller \\
$\eta_{p}$ & 0.80 & efficiency of motor \\
$\eta_{m}$ & 0.90 & efficiency of speed controller \\
$\eta_{e s c}$ & 0.95 & sink rate polar fit in $\frac{\mathrm{m} / \mathrm{s}}{v_{a}}$ \\
a,b,c & $-0.0059,0.1507,-1.4833$ & \\
\hline \hline
\end{tabular}

Table 6. Summary of parameters used in behavior determination

\begin{tabular}{cc}
\hline \hline parameters & description \\
\hline$\Delta t_{\text {plan }}=1$ sec & interval for aircraft's mode determination \\
$\Delta i_{\text {local }}=3$ & number of columns above and below current cell \\
$\Delta j_{\text {local }}=3$ & number of rows above and below current cell \\
$\Delta i_{\text {global }}=30$ & number of columns above and below current cell \\
$\Delta j_{\text {global }}=30$ & number of rows above and below current cell \\
\hline \hline
\end{tabular}

Table 7. Miscellaneous parameters used in this paper

\begin{tabular}{ccr}
\hline \hline variable & value & description \\
\hline $\mathrm{r}$ & 30 meters & steady turn radius for thermal centering controller \\
$\mathrm{k}$ & 25 & scale factor to reflect importance of energy acceleration \\
\hline \hline
\end{tabular}




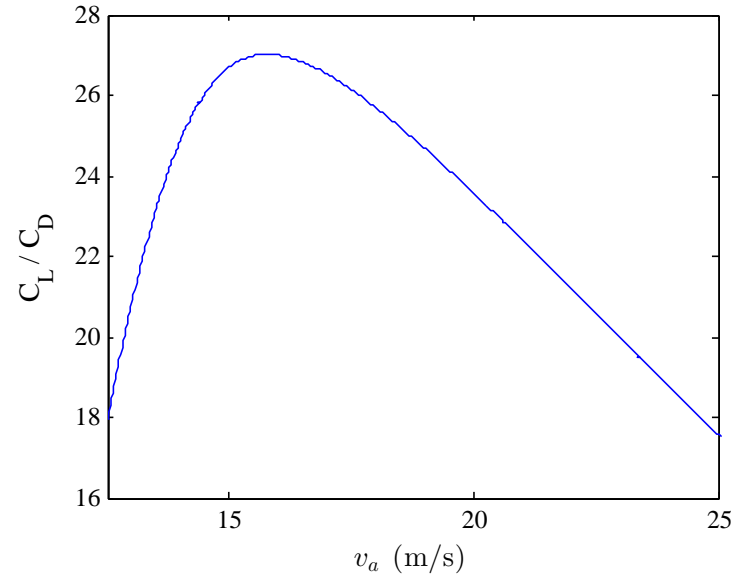

(a) L/D over a range of airspeed

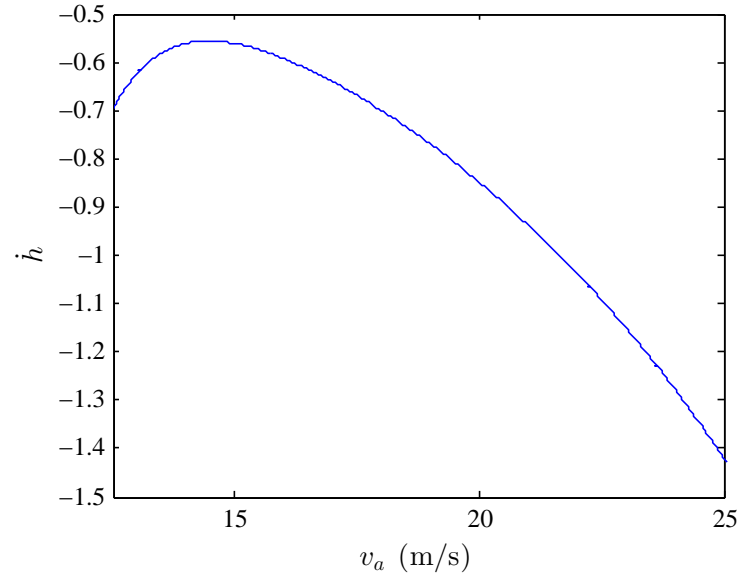

(b) Sink rate polar

Figure 15. SB-XC aircraft model 